\title{
Nanophotonic Reservoir Computing for Noisy Time Series Classification
}

\author{
M. R. Salehi, E. Abiri, and L. Dehyadegari
}

\begin{abstract}
Reservoir computing is known as a recent training concept in machine learning. This method is particularly useful in solving a broad category of categorization and recognition problems. The aim of this paper is using photonic reservoir computing for noisy time series classification. A complex network of photonic crystal cavities is used for modeling photonic reservoir computing. Applying nanophotonic reservoir computing resulted in perfect $(\mathbf{1 0 0 \%})$ recognition accuracy for noise-less time series classification, and an accuracy of about 98\% for noisy time series, which shows improvement (an amount of $3 \%$ ) compared to previous works.
\end{abstract}

Index Terms - Nanophotonic reservoir computing, time series classification, photonic crystal cavities.

\section{INTRODUCTION}

Reservoir computing is a newly proposed method [1]-[3] in the field of machine learning which is a recurrent neural network with sparse and random weights. As an instant, these networks are used in classification problems such as dynamic pattern recognition [3], chaotic time series classification and recognition [4], speech recognition [5]-[7] and noise modeling [4].

Photonic reservoir computing is a platform for reservoir computing using photonics technology. A network of interconnected optical structures can outperform electronics in speed, bandwidth and power use [8]. A nanophotonic reservoir with coupled nonlinear photonic crystal cavities reveals a rich dynamical behavior and can be made use of to perform optical signal processing.

Due to unique nonlinear effects, small size, high speed and very low energy consumption, photonic crystal cavities are good candidates for all cases of optical reservoir computing. High Q-factor photonic crystal cavities enable greater interaction with the matter and result in more interesting dynamics. They can start to self-pulsate due to generation of free carriers and changing of the refract index [9], [10]. Thereby we will demonstrate how these properties emulate the behavior of spiking neurons on a photonic chip [11].

This paper aims to model reservoir computing scheme using photonic crystal cavities and also intends to study the function of this structure on noisy time series recognition. Section II below describes photonic reservoir computing in

Manuscript received November 3, 2013; revised March 5, 2014

M. R. Salehi is with the Department of Electrical and Electronics Engineering, Shiraz University of Technology (Sutech), Modarres Boulevard, P.O, Box 16315-1618, Shiraz, Iran (e-mail: salehi@sutech.ac.ir; tel: +98 7117353500 ; fax: +98 7117354514$)$.

E. Abiri and L. Dehyadegari are with the Department of Electronics, Shiraz University of Technology, P.O, Box 16315-1618, Shiraz, Iran (e-mail abiri@sutech.ac.ir, 1.dehyadegari@sutech.ac.ir). detail and demonstrates coupled mode theory (CMT) equations for modeling photonic crystal cavities as reservoir nodes. Time series categorization and obtained results will be discussed in Section III, and finally Section IV summarizes the main findings of this paper.

\section{PHOTONIC RESERVOIR COMPUTING}

Photonic reservoir computing is a photonic circuit which is a hardware implementation of reservoir networks. Nonlinear optical components provide a very energy-efficient and rich dynamic for classification and recognition problems.

\section{A. Principles of Reservoir Computing}

Neural networks are an example of human computational power, and reservoir computing is a type of recurrent neural networks which avoids the problems of training weights [8]. In reservoir computing framework, a randomly fixed recurrent neural network that is left untrained is excited with an external stimulus. A nonlinear mapping of the input signal into a high dimensional feature space is essentially performed by the reservoir. The readout function maps the resulted feature space to the desired output by a simple linear regression function [12]

Fig. 1 shows the reservoir which consists of reservoir, readout and its input and output.

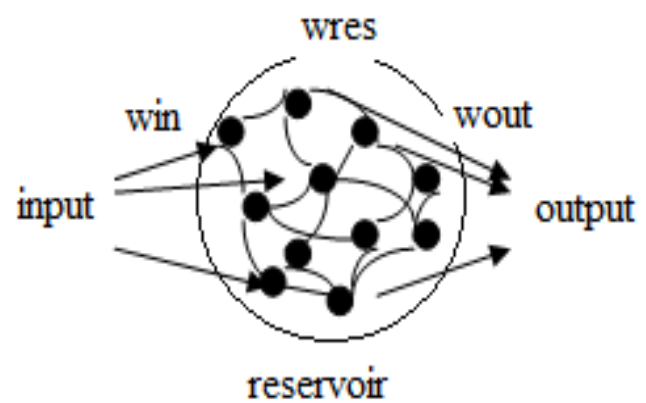

Fig. 1. Reservoir nodes and its input and output, input signals are fed into the reservoir nodes; then, a nonlinear mixing of the input in the feature space is performed and the output will be the state of reservoir nodes.

Eq. 1 shows the reservoir dynamics:

$$
x(t+\Delta t)=(1-\eta) x(t)+\eta f\left(w_{i n} u(t)+w_{r e s} x(t)\right)
$$

where $p_{\text {out }}$ and $\varphi_{\text {out }}$ are output power and phase of the reservoir, $p_{\text {in }}$ and $\varphi_{\text {in }}$ are input power and phase, $h(\tau)$ shows the gain $g$ integrated over the length $L$ of the amplifier, $\tau_{c}$ and $E_{s a t}$ show carrier lifetime and gain saturation, $\beta_{c}$ is line width enhancement factor and $g_{0}$ indicates small signal gain [13]. 
Since the training of weights is limited to the readout function, it is much simpler than that of other neural networks to have a hardware implementation of this structure. Nanophotonics provides us with some candidates for this purpose such as optical amplifiers [5], [14], lasers and photonic crystal cavities. Nonlinear characteristics, dynamic behavior, very energy efficient and high speed lend photonic crystal cavities a complex behavior for implementation of reservoir computing.

\section{B. Photonic Crystal Cavities Simulation Model}

CMT equations have been used for modeling photonic crystal cavities [10], [11]. This model captures basic features such as the characteristic power of a cavity, carrier density, temperature, resonance frequency of the cavity, phase difference between two cavities and detuning from the resonant frequency.

The CMT equations are then [15]:

$$
\begin{gathered}
\frac{d a}{d t}=\left[j\left(\omega_{r}+\delta \omega_{n l}-\omega\right)-\frac{\gamma_{l o s s}}{2}\right] a+k s_{i n} \\
\frac{d \Delta T}{d t}=-\frac{\Delta T}{\tau_{t h}}+\frac{\Gamma_{t h^{\prime}}{ }_{a b s}|a|^{2}}{\rho_{s i}{ }_{p, s i} V_{t h}} \\
\frac{d N}{d t}=-\frac{N}{\tau_{f c}}+\frac{\Gamma_{F C A} \beta_{s i} c^{2}|a|^{4}}{2 \hbar \omega V_{F C A}^{2} n g^{2}} \\
s_{\text {out }}=e^{j \varphi_{c}}{ }_{s_{\text {in }}}+k a
\end{gathered}
$$

where $a=|a| e^{j \varphi}$, with $|a|^{2}$ the energy in the cavity and $\varphi$ the phase, $s_{\text {in }}$ and $s_{\text {out }}$ are the amplitudes of the input and output lights, $\Delta T$ is temperature difference with the surroundings, $N$ shows the amount of free carriers, $k$ is the coupling from waveguide to ring, $\varphi_{c}$ indicates phase propagation in the bus waveguide, $\omega$ and $\omega_{r}$ are the resonance frequency of the cavity and the frequency of the input light respectively, $\tau_{f c}$ and $\tau_{\text {th }}$ are relaxation times for free carriers and temperature, $\beta_{s i}$ is a constant governing two photon absorptions, $n_{g}$ is the group index, $\rho_{s i}$ indicates the density of the silicon, $c_{p, s i}$ shows the thermal capacity, effective volumes $V_{\alpha}$ and confinements $\Gamma_{\alpha}$ corresponding with a physical effect $\alpha$ and $\gamma_{\text {abs }}$ and $\gamma_{\text {loss }}$ are absorption and total losses in the cavity.

Numerical methods such as Rung-Kutta4 can be used to solve the CMT equations. To obtain the steady state curves, we keep the input wavelength as fixed and put the derivative of Eq. 2 equal to zero, and then simply parameterize $\Delta T, N$ and $P_{\text {in }}$ as a function of $|a|^{2}$. Table I shows the parameter values.

As shown in the results (Fig. 2 and Fig. 3), the difference between slow heating effects and the fast free carrier dynamics causes self-pulsation (Fig. 2) and excitability (Fig. 3 ) in photonic crystal cavities. This nonlinear behavior suggests a network of photonic crystal cavities as a nanophotonic reservoir to perform optical signal processing.
TABLE I: PARAMETER VALUES THAT USED IN SIMULATIONS

\begin{tabular}{lcc}
\hline \hline Parameter & Value & Magnitude \\
\hline$\beta_{s i}$ & $8.4^{*} 10^{-12}$ & $\mathrm{~m} \cdot \mathrm{W}^{-1}$ \\
$\sigma_{s i}$ & $10^{-21}$ & $\mathrm{~m}^{2}$ \\
$\rho_{s i}$ & 2.33 & $\mathrm{~g} \cdot \mathrm{m}^{3}$ \\
$c_{p, s i}$ & 0.7 & $\mathrm{~J} \cdot \mathrm{g}^{-1} \cdot \mathrm{K}^{-1}$ \\
$n_{g}=n_{s i}$ & 3.476 & \\
$\lambda_{r}$ & 1552.77 & $\mathrm{~nm}$ \\
$\tau_{t h}$ & 65 & $\mathrm{~ns}$ \\
$\tau_{f c}$ & 5.3 & $\mathrm{~ns}$ \\
$\Gamma_{t h}$ & 0.9355 & \\
$\Gamma_{T P A}$ & 0.9964 & \\
$\Gamma_{F C A}$ & 0.9996 & \\
$V_{t h}$ & 3.19 & $\mu m^{3}$ \\
$V_{T P A}$ & 2.59 & $\mu m^{3}$ \\
\hline \hline
\end{tabular}
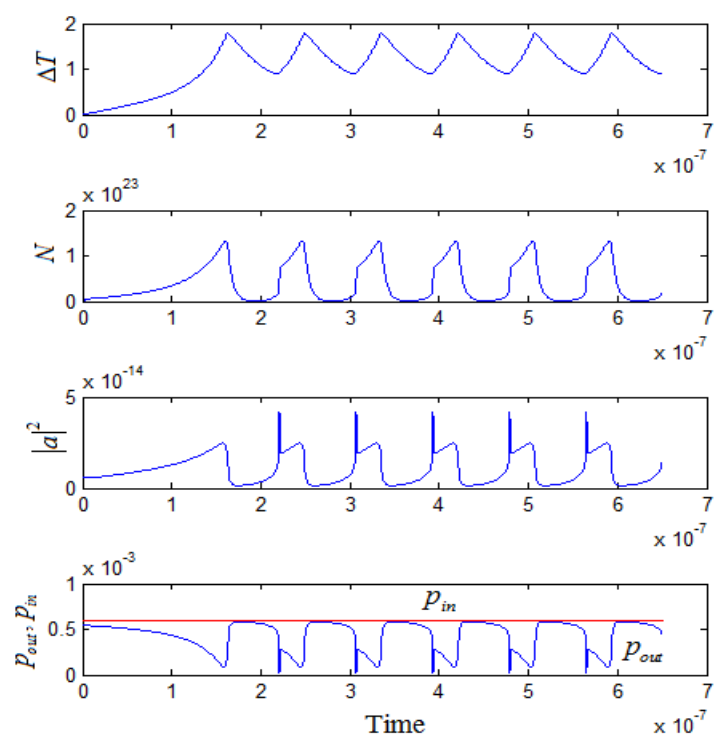

Fig. 2. Results of simulations with CMT equations; in input wavelength $\lambda=1550 \mathrm{~nm}$ and input power $p_{\text {in }}=0.6 \mathrm{mw}$ the photonic crystal cavity self-pulsates.
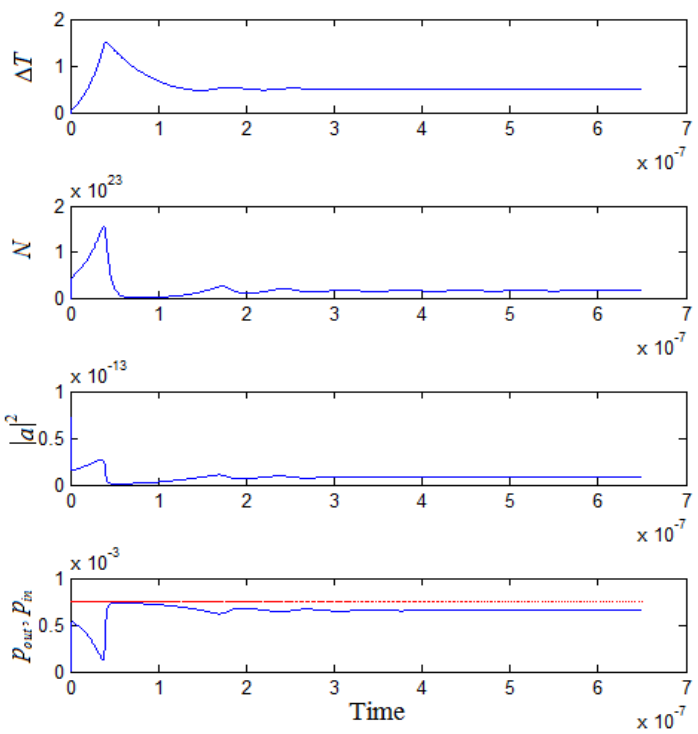

Fig. 3. Results of simulations with CMT equations; in input wavelength $\lambda=1550 \mathrm{~nm}$ and input power $p_{\text {in }}=0.7 \mathrm{mw}$ the photonic crystal cavity is excitable. 


\section{TIME SERIES CLASSIFICATION}

A simple but non-partial noisy signal classification task was used in the simulation as in previous works [16], [17]. The time series have random switches between square and triangular wave forms. These two different time series were required to be classified and the output signal followed the inputs changes as fast as possible.

For better comparison, a reservoir with 25 nodes and waterfall topology [8] was drown on in experiments as used in earlier works [16], [17].

Fig. 4a shows an example of time series with its desired output while Fig $4 \mathrm{~b}$ demonstrates reservoir state vectors (output power of photonic crystal cavities) for this time series, and Fig. $4 \mathrm{c}$ shows the output results of the readout and the final output by applying the sign function.

The fraction of time in which the reservoir gives a correct classification is defined as recognition accuracy. Photonic reservoir computing with a network of photonic crystal cavities resulted in $100 \%$ recognition accuracy for noise-less time series.
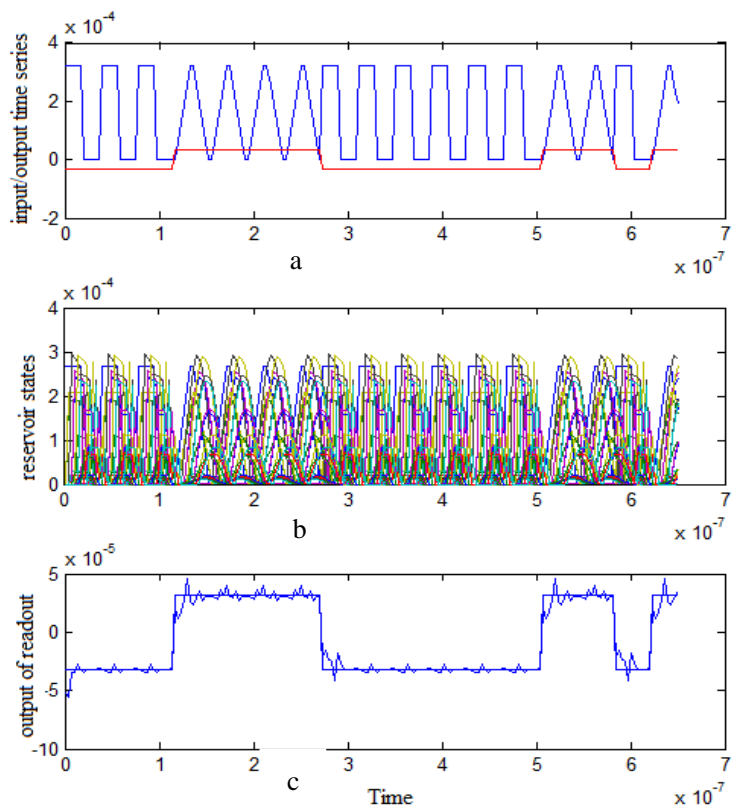

Fig. 4. One of the input time series for reservoir (input power of the photonic crystal cavities), the reservoir state (output power of the photonic crystal cavities) and output signal of photonic readout.

\section{NOISY TIME SERIES ClassifiCATION}

Noisy time series with different signal-to-noise ratios were used in this stage for classification. Fig. 5a shows an example of noisy time series with $20 \mathrm{db}$ of signal-to-noise ratio and its desired output. Fig 5 b shows reservoir state vectors and Fig. $5 \mathrm{c}$ demonstrates output results from the readout function. A White noise with different signal-to-noise ratios was used and the recognition accuracy was compared with previous works, as observed in Fig. 6.

Small size, high speed and very low energy consumption caused photonic crystal cavities good candidates for modelling optical reservoir computing. High Q-factor photonic crystal cavities are self-pulsate and were investigated for hardware implementation of reservoir computing.
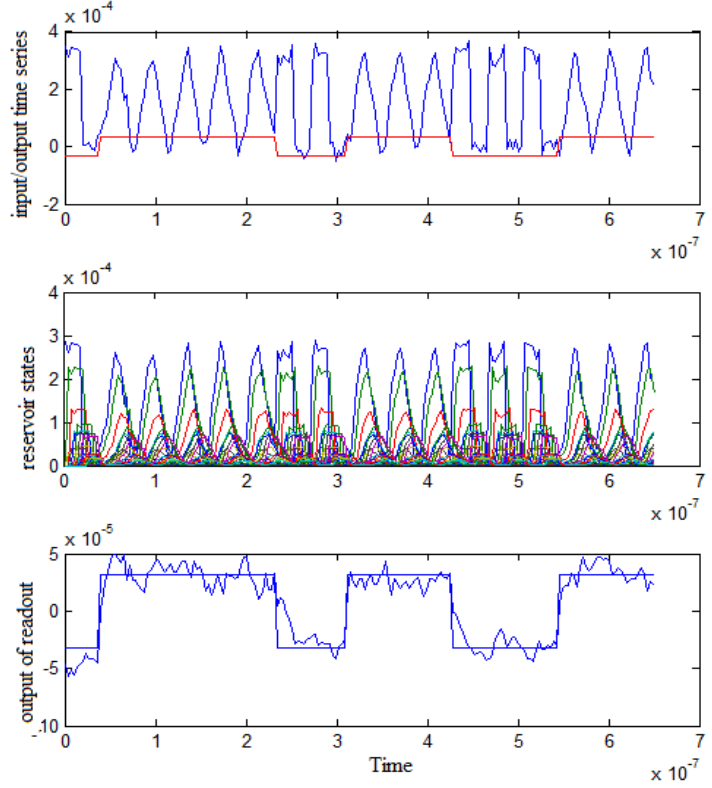

Fig. 5. One of the noisy input time series for reservoir with $20 \mathrm{db}$ of signal to noise ratio (input power of the photonic crystal cavities), the reservoir state (output power of the photonic crystal cavities) and output signal of photonic readout.

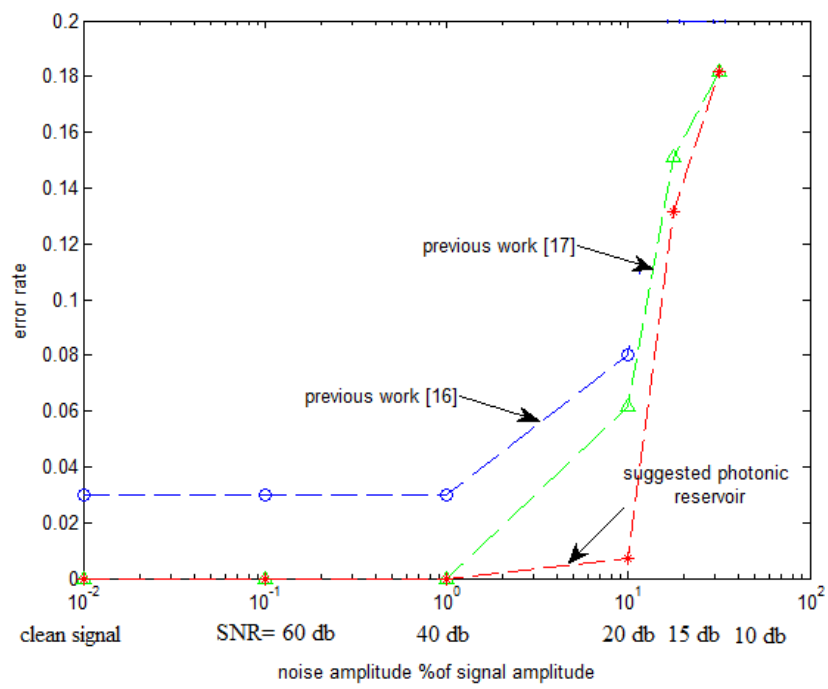

Fig. 6. Classification error rates for different signal to ratios. The results are compared with the previous works

\section{CONCLUSION}

In this paper a nanophotonic reservoir was drown on to perform optical signal processing. Unique nonlinear effects, The application of photonic crystal cavities as reservoir nodes resulted in perfect recognition accuracy for the time series without noise. For noisy time series with 20 to $60 \mathrm{db}$ of signal-to-noise ratios the recognition accuracy varied between $99.82 \%$ to $100 \%$ which shows about $7 \%$ improvement over the previous work. These advantages suggested photonic reservoir computing with a network of photonic crystal cavities as a good candidate for other classification tasks and large signal processing, such as speech recognition.

\section{REFERENCES}

[1] W. Maass, T. Natschlager, and H. Markram, "Real-time computing without stable states: A new framework for neural computation based on perturbations," Journal of Neural Computing, vol. 14, pp. 2531-2560, 2002. 
[2] L. Buesing, J. Bill, B. Nessler, and W. Maass, "Neural dynamics as sampling: a model for stochastic computation in recurrent networks of spiking neurons," Journal of PLoS Comput Biol, vol. 7, pp. 1-22, 2011.

[3] H. Jaeger and H. Haas, "Harnessing nonlinearity: predicting chaotic systems and saving energy in wireless communication," Journal of Science, vol. 304, pp. 78-80, 2004

[4] Y. Paquot, J. Dambre, B. Schrauwen, M. Haelterman, and S. Massar, "Reservoir computing: a photonic neural network for information processing," Journal of Nonlinear Optics and Applications IV, vol. 7728, pp. 1-33, 2010.

[5] K. Vandoorne, J. Dambre, D. Verstraeten, B. Schrauwen, and P. Bienstman, "Parallel reservoir computing using optical amplifiers," IEEE Trans. Neural Netw, vol. 22, pp. 1469-1481, 2011.

[6] K. Vandoorne, M. Fiers, D. Verstraeten, B. Schrauwen, J. Dambre, and P. Bienstman, "Photonic reservoir computing: a new approach to optical information processing," in Proc. Transparent Optical Networks Conference, 2010, vol. 12, pp. 1-4.

[7] D. Verstraeten, B. Schrauwen, and D. Stroobandt, "Reservoir based techniques for speech recognition," in Proc. the IEEE International Joint Conference on Neural Networks, 2006, pp. 1050-1053.

[8] K. Vandoorne, "Photonic reservoir computing with a network of coupled semiconductor optical amplifiers," Ph.D. dissertation, Gent University, 2011

[9] T. V. Vaerenbergh, M. Fiers, P. Mechet, T. Spuesens, R. Kumar, G. Orthier, B. Schrauwen, J. Dambre, and P. Bienstman, "Cascadable excitability in microrings," Journal of Optics Express, vol. 20, pp. 20292-20308, 2012.

[10] T. V. Vaerenbergh, M. Fiers, J. Dambre, and P. Bienstman, "Simplified description of self-pulsation and excitability by thermal and free-carrier effects in semiconductor microcavities," Journal of Physical Review, vol. 86, pp. 063808-1 - 063808-8, 2012.

[11] M. Fiers, B. Maes, and P. Bienstman, "Dynamics of coupled cavities for optical reservoir computing," in Proc. Symposium IEEE Photonics Benelux Conference, Brussels, 2009, vol. 14, pp. 129-132.

[12] Y. Paquot, F. Duport, A. Smerieri, J. Dambre, B. Schrauwen, M. Haelterman, and S. Massar, "Optoelectronic reservoir computing," Journal of Scientific Reports, vol. 2, pp. 1-6, 2012.

[13] M. Lukoševicius, H. Jaeger, and B. Schrauwen, "Reservoir computing trends," Journal of Künstl Intell, vol. 26, pp. 365-371, 2012.

[14] M. R. Salehi, E. Abiri, and L. Dehyadegari, "Analytical approach in photonic reservoir computing a network of SOA for noisy speech recognition," Journal of Optics Communication, vol. 306, pp. 135-139, 2013.

[15] T. J. Johnson, M. Borselli, and O. Painter, "Self-induced optical modulation of the transmission through a high-Q silicon microdisk resonator," Jounal of Opt. Express, vol. 14, pp. 817-831, 2006.
[16] K. Vandoorne, W. Dierckx, B. Schrauwen, D. Verstraeten, R. Baets, P. Bienstman, and J. V. Campenhout, "Toward optical signal processing using photonic reservoir computing," Journal of Opt. Exp., vol. 16, no. 15, pp. 11182-11192, 2008.

[17] M. R. Salehi and L. Dehyadegari, "Analytical approach for noisy time series classification using photonic reservoir computing," presented at $4^{\text {th }}$ International Conference on Electrical Electronics and Civil Engineering, 2013.

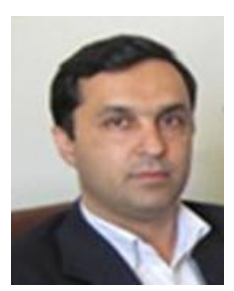

Mohammad Reza Salehi received the B.Sc. degree in electrical engineering from Amirkabir University of Technology (Tehran Polytechnique), Tehran, the M.Sc. degree in electrical engineering from Shiraz University, Shiraz, Iran, and the Ph.D. degree in optoelectronics at the ENSERG/INPG, France. He has authored and coauthored over 85 journal and conference papers and 7 books. He is currently a member of IEEE. His research interests include optoelectronics, optomicrowaves and optical systems.

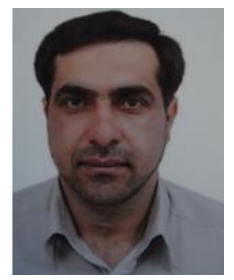

Ebrahim Abiri received the B.Sc. degree in electronics engineering from Iran University of Science and Technology (IUST) in 1992, M.Sc. degree from Shiraz University in 1996 and the Ph.D. degree in electronic from Iran University of Science and Technology (IUST) in 2007. He has authored more than 50 published technical papers in electronics and power electronics and 4 books. He has been with the Department of Electrical Engineering, Shiraz University of Technology (SUTECH) since 2007. His current research activities include analog circuit design and power electronic.

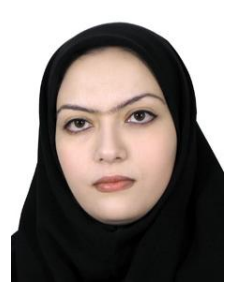

Louiza Dehyadegari received her B.Sc. degrees in biomedical engineering from University of Isfahan, Isfahan, Iran in 2003 and M.Sc. degrees in Amirkabir University of Technology (Tehran Polytechnic), Tehran, Iran, in 2005. She currently continues her $\mathrm{Ph} . \mathrm{D}$ in electronic engineering at the Shiraz University of Technology. Her research interests focus on artificial neural networks, speech recognition, optical signal processing, photonic neural networks and photonic reservoir computing. 